\title{
Effectiveness and safety of PD-1/PD-L1 or CTLA4 inhibitors combined with chemotherapy as a first-line treatment for lung cancer: A meta-analysis
}

\author{
Kaikai Shen ${ }^{1 \#}$, Jinggang Cui ${ }^{2 \#}$, Yuqing Wei ${ }^{3}$, Xiaojun Chen ${ }^{2}$, Guohua Liu ${ }^{2}$, Xiaolai Gao ${ }^{2}, \mathrm{Wei}^{2}$, \\ Huiling Lu ${ }^{2}$, Ping Zhan ${ }^{3}$, Tangfeng Lv ${ }^{3}$, Dang Lin ${ }^{2}$ \\ ${ }^{1}$ Wannan Medical College, Wuhu 241001, China; ${ }^{2}$ Department of Respiratory Medicine, Suzhou Hospital Affiliated Nanjing Medical University, 16 \\ West BAITA Road, Suzhou 215001, China; ${ }^{3}$ Department of Respiratory and Critical Care Medicine, Jinling Hospital, Medical School of Nanjing \\ University, Nanjing 210002, China \\ Contributions: (I) Conception and design: K Shen, J Cui; (II) Administrative support: D Lin; (III) Provision of study materials or patients: K Shen, Y \\ Wei; (IV) Collection and assembly of data: K Shen, Y Wei; (V) Data analysis and interpretation: K Shen, Y Wei, J Cui; (VI) Manuscript writing: All \\ authors; (VII) Final approve of manuscript: All authors. \\ \#These authors contributed equally to this work. \\ Correspondence to: Dang Lin. Department of Respiratory Medicine, Suzhou Hospital Affiliated Nanjing Medical University, 16 West BAITA Road, \\ Suzhou 215001, China. Email: Danglin4067@163.com.
}

Background: Immune checkpoint inhibitors (ICIs) combined with chemotherapy have been applied as a first-line treatment for lung cancer, but consistent beneficial results have not been documented. Therefore, our meta-analysis aimed to evaluate the effectiveness and safety of combination therapy to promote its application.

Methods: We searched electronic databases for studies that estimated the safety and efficacy of combined therapy. The objective response rate (ORR) and disease control response (DCR) parameters were evaluated with odds ratio (OR) values of the combination arm over the non-combination arm. Hazard ratios (HR) and its $95 \%$ confidence intervals (95\% CI) were used to calculate progression-free survival (PFS) and overall survival (OS) in the combination and non-combination arms. All treatment-related adverse events (TRAEs) and 3 to 5 TRAEs were expressed as relative risk (RR) values of the combination arm over the noncombination arm.

Results: Ten eligible studies involving 4,887 patients were identified. The pooled ORs for ORR and DCR were 1.85 (95\% CI: 1.30-2.63, $\mathrm{P}<0.01$ ) and 1.14 (95\% CI: 0.70-1.86, $\mathrm{P}<0.01$ ), respectively. The pooled HRs for PFS and OS were 0.67 (95\% CI: 0.58-0.79, P<0.001) and 0.76 (95\% CI: 0.65-0.88, P<0.001), respectively. In subgroup analysis, ORR and DCR were significantly improved in the programmed cell death-1/L1 (PD-1/L1) blockade for non-small cell lung cancer (NSCLC) group (subgroup A), with a combined OR values of 2.36 (95\% CI: 1.79-3.13, P<0.001) and 1.92 (95\% CI: 1.10-3.35, P<0.001), respectively. However, no significant benefits were observed in the cytotoxic $\mathrm{T}$ lymphocyte antigen-4 (CTLA-4) blockade for small cell lung cancer (SCLC) (subgroup B) and CTLA-4 blockade for NSCLC groups (subgroup C). In addition, a significant improvement in PFS was observed in subgroup A, subgroup $\mathrm{B}$ and subgroup $\mathrm{C}$, with pooled HR values of 0.58 (95\% CI: 0.52-0.63, P<0.001), 0.86 (95\% CI: 0.76-0.97, $\mathrm{P}<0.05$ ) and 0.83 (95\% CI: 0.68-1.00, $\mathrm{P}<0.05$ ), respectively. Only subgroup A exhibited an OS benefit, with a combined HR value of 0.67 (95\% CI: 0.55-0.81, P<0.001). Moreover, as the expression of PD-L1 increased, the PFS and OS benefits were more significantly. Furthermore, patients without central nervous system (CNS) metastasis who were treated with $\mathrm{PD}-1 / \mathrm{L} 1$ inhibitors had a longer OS than patients with CNS metastasis (HR: 0.67, 95\% CI: 0.55-0.80, P<0.001). Finally, combined therapy was associated with 3 to 5 TRAEs (RR: 1.26, 95\% CI: $1.08-1.47 ; \mathrm{P}<0.01)$.

Conclusions: Patients treated with immunotherapy and chemotherapy in combination exhibited superior 
in ORR, DCR, PFS and OS as well as slightly increased TRAE levels compared with those of patients treated with either monotherapy.

Keywords: Lung cancer, immunotherapy; PD-1/L1 inhibitors; CTLA-4 inhibitors; chemotherapy

Submitted Jun 10, 2018. Accepted for publication Nov 15, 2018.

doi: $10.21037 /$ jtd.2018.11.72

View this article at: http://dx.doi.org/10.21037/jtd.2018.11.72

\section{Introduction}

Lung cancer is a malignant tumor that exhibits among the highest morbidity and mortality rates worldwide, and 57\% of lung cancer patients are in advanced stages of disease at the time of first diagnosis. Non-small cell lung cancer (NSCLC) accounts for approximately $80 \%$ to $85 \%$ of all lung cancer cases and has a 5 -year survival rate of less than $15 \%$ (1). Platinum-based traditional chemotherapy has long been the standard first-line treatment for lung cancer and has objective response rates (ORRs) ranging from 15-32\% (2). Currently, lung cancer treatment remains difficult worldwide. Unfortunately, despite the emergence of targeted therapies and the improvement of traditional treatments, disease progression and drug resistance remain unavoidable.

With the development of immunotherapy and an increased understanding of tumor immunity, immunotherapy has gradually been applied in the clinic. Immune checkpoint inhibitors (ICIs) can remove the inhibitory signals between tumor cells and $\mathrm{T}$ cells and reactivate $\mathrm{T}$ cell immune responses (3). Moreover, some clinical trials have demonstrated that immunotherapy can dramatically improve the prognosis of lung cancer patients compared better than standard chemotherapy alone $(4,5)$. Current, immunotherapy regimens mainly include programmed cell death-1 (PD-1) (nivolumab or pembrolizumab), programmed cell death-L1 (PD-L1) (atezolizumab, durvalumab or avelumab) and cytotoxic T lymphocyte antigen-4 (CTLA-4) (ipilimumab) ICIs. $\mathrm{PD}-1 / \mathrm{L} 1$ inhibitors are considered to be very promising drugs in the treatment of NSCLC. Recently, nivolumab was approved by the US Food and Drug Administration (FDA) for the treatment of recurrent metastatic NSCLC with targeted drugs or in combination with platinumbased chemotherapy, and pembrolizumab was approved for NSCLC as a second-line or higher treatment in combination with PD-L1 and as a first-line treatment for advanced NSCLC (6). CTLA-4 was the earliest known immune checkpoint and exclusively expressed in $\mathrm{T}$ cells, thereby inhibiting the $\mathrm{T}$ cell response when it attaches to B7 on antigen-presenting cells (APCs) (7). In addition, CTLA-4 inhibitors enhance the immune system by releasing checkpoints and removing regulatory $\mathrm{T}$ cells from the tumor via an antibody-dependent cytotoxic mechanism (8). In fact, single-agent PD-1/L1 blockade therapy has limited activity, exhibiting ORRs of $15-25 \%$ (9). New research results demonstrate that chemotherapeutic agents can augment antitumor immunosurveillance and subsequently enhance effector T cell activity (10). Therefore, immunotherapy combined with chemotherapy has offered new breakthroughs in lung cancer treatment, and a considerable number of similar treatments have been researched in clinical environments. Currently, few studies have demonstrated that immunotherapy combined with chemotherapy can synergistically improve the prognosis of patients with lung cancer $(11,12)$. However, recently published studies evaluating the survival benefits of combination therapy versus chemotherapy alone showed that combination therapy did not significantly benefit survival, and the rates of some treatment-related adverse events (TRAEs) were even increased $(11,13)$. Furthermore, some lung cancer patients developed hyperprogressive disease (HPD) and exhibited fatal adverse reactions after receiving immunotherapy $(14,15)$. Therefore, it is important to weigh the benefits and risks between efficacy and adverse reactions. Our meta-analysis provides a basis for the assessment of immunotherapy combined with chemotherapy and provides evidence supporting a new therapeutic regimen for lung cancer patients.

\section{Methods}

\section{Search strategy and study selection}

Electronic databases, including PubMed, Embase, and Web of Science, were searched for studies published from 2008 to 2018 that could be included in this meta- 
analysis. A computerized search of the online proceedings of the ASCO Annual Meeting, the European Society for Medical Oncology (EMSO) and the Word Conference of Lung Cancer (WCLC) was also performed. The dates of the search ranged from the inception of each database to October 10, 2018. Two investigators (Kaikai Shen and Yuqing Wei) independently searched the databases. The following keywords were used: (immunotherapy OR PD-1/PD-L1 inhibitors OR CTLA-4 inhibitors OR programmed death-ligand 1 OR programmed death-ligand L1 OR pembrolizumab OR nivolumab OR atezolizumab OR ipilimumab OR durvalumab [Title/Abstract]) AND (chemotherapy [Title/Abstract]) AND (lung cancer OR lung tumor OR lung neoplasms OR NSCLC [Title/ Abstract]). Finally, the search was extended by reviewing references included in the retrieved articles. Study selection was based on the Preferred Reporting Items for Systematic Reviews and Meta-Analyses (PRISMA) statement (16). This study was approved by the ethics committee of Jinling Hospital affiliated to Medical School of Nanjing University.

\section{Inclusion criteria and exclusion criteria}

The following predetermined inclusion criteria were used: (I) the study reported the use of PD-1/L1 inhibitors or an anti-CTLA4 antibody combined with chemotherapy for the treatment of lung cancer; (II) the study directly or indirectly investigated any of the following measurements: ORR, disease control response (DCR), progression-free survival (PFS), overall survival (OS) and incidence of TRAEs; (III) the study assessed first-line lung cancer treatments; (IV) the study type was a randomized clinical trial (RCT); and (V) the study was published in English. The following predetermined exclusion criteria were used: (I) studies without related data; (II) letters, case reports, and reviews; and (III) duplicate publications.

\section{Data extraction}

Two independent authors (Kaikai Shen and Yuqing Wei) extracted the data from the eligible studies. Disagreements were resolved by discussion or consensus with a third reviewer (Jinggang Cui). The following data were included: authors, publication year, study type, patient numbers, randomization, trial phase, treatment strategy, pathological type, events of complete response, partial response, stable disease $v s$. progressive disease, PFS, OS, and incidence of any grade and grade 3 to 5 TRAEs.

\section{Statistical analysis}

Relevant data were extracted from each study, and the pooled odds ratio (OR) for ORR and DCR, hazard ratio (HR) for PFS and OS, and relative risk (RR) for any grade and grade 3 to 5 TRAEs were estimated via a meta-analysis. $95 \%$ confidence interval (95\% CI) was used for HR endpoint values. Heterogeneity was assessed using $\mathrm{Chi}^{2}$ testing and the $\mathrm{I}^{2}$ statistic. A $\mathrm{P}$ value less than 0.05 indicates significant heterogeneity, and an $\mathrm{I}^{2}$ value greater than $50 \%$ indicates significant heterogeneity (17). Visual inspection of the funnel plot and Egger's linear regression test were performed to assess publication bias. A fixed effects model was used in the analyses in which no substantial heterogeneity was noted among the studies; otherwise, the random effects model was applied. All the statistical tests used in our meta-analysis were performed using STATA version 12.0 software (Stata Corporation College Station, TX, USA). For all analyses, a P value less than 0.05 indicates statistical significance.

\section{Quality assessment}

The methodological quality of randomized trials was assessed using the Cochrane risk of bias tool (18). Two reviewers (Kaikai Shen and Yuqing Wei) evaluated the risk of bias for all the included studies by random sequence generation, allocation concealment, blinding of participants and personnel, blinding of outcome assessment, incomplete outcome data, selective reporting and other biases. Each item of bias was classified as three levels: "Yes" for a low risk of bias, "No" for a high risk of bias and "Unclear". Any disagreements on judgment of the high, low or unclear risks were resolved by a third investigator (Jinggang Cui). Finally, the bias risks were categorized into three levels: low risk, high risk and unclear.

\section{Results}

\section{Search results and characteristics of the included studies}

The screening process for study inclusion was summarized in Figure 1. A total of 507 results were retrieved from PubMed, 857 results were retrieved from Web of Science, 419 results were retrieved from Embase and 3 results were retrieved from other sources (WCLC, ASCO and ESMO). A total of 423 duplicated records were excluded. After referring to full texts or abstracts, 310 records were removed that did not satisfy the inclusion criteria, and 23 studies did not provide the relevant outcome. Ten studies involving three 


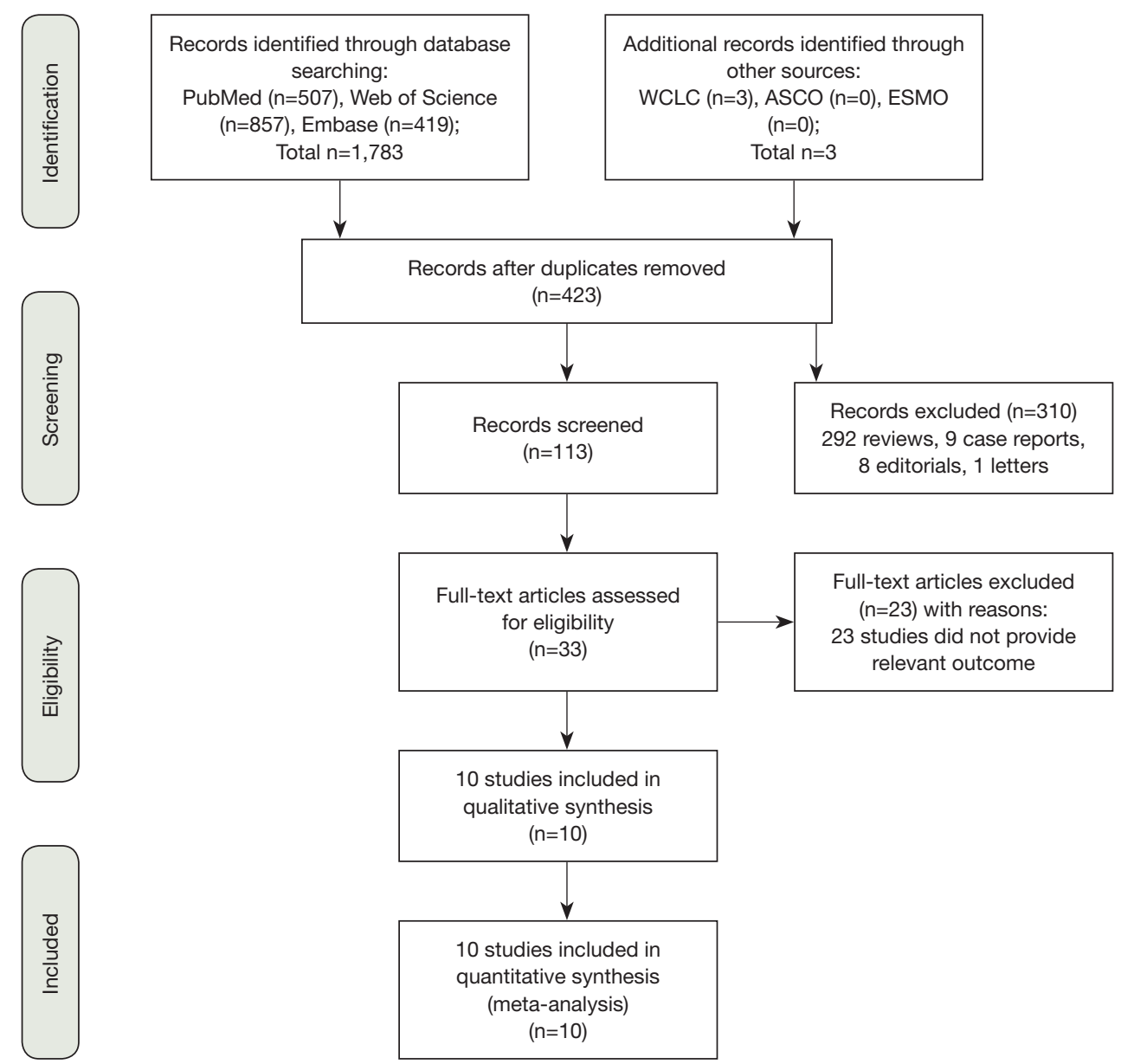

Figure 1 Flow-chart of the different steps in the meta-analysis.

phases 2 trials (19-21) and seven phase 3 trials (13,22-27) were identified for inclusion in our meta-analysis. All of the studies were RCTs and most trials were in phase 3 . All studies involved first-line treatments of advanced lung cancer patients. Regarding pathological types, seven studies involved NSCLC, and three involved SCLC. Moreover, of the seven NSCLC trials, three involved non-squamous NSCLC, and two involved squamous NSCLC. Seven studies were two-armed trials $(13,19,22-24,26,27)$, and three studies were triple-armed trials $(20,21,25)$. In addition, in the two triple-armed studies $(20,21)$, data related to the phase dosing regimen were selected for assessment in our meta-analysis. Five articles (19,24-27) and one article (13) investigated PD-1/L 1 inhibitors combined with chemotherapy in NSCLC and SCLC patients, respectively, and two articles $(20,22)$ and two articles $(21,23)$ investigated CTLA-4 inhibitors combined with chemotherapy in SCLC and NSCLC patients, respectively. Ten and eight studies directly or indirectly provided data to calculate the ORs for ORRs and DCRs, respectively. The HR values and its 95\% CIs for PFS and OS were available for extraction in all trials. However, the HR values and its $95 \%$ CIs for PFS and OS related to PD-L1 expression levels $<1 \%, 1-49 \%$ and $\geq 50 \%$ were available for extraction in only four and two studies, respectively. The HR value and its $95 \%$ CI for OS on brain metastases were available in only four studies. Furthermore, all studies were available to obtain RR for any grade TRAEs and grade 3 to 5 TRAEs. In total, 4,887 patients were included in our meta-analysis. All of the study patients had a PS of 0 to 1 . The median age ranged from 61 to 65 years old. Detailed characteristics of the study were summarized in Table 1.

\section{The risk of methodological bias}

The study qualities were evaluated by risk of bias assessment. 
Table 1 Characteristics of all studies included in the meta-analysis

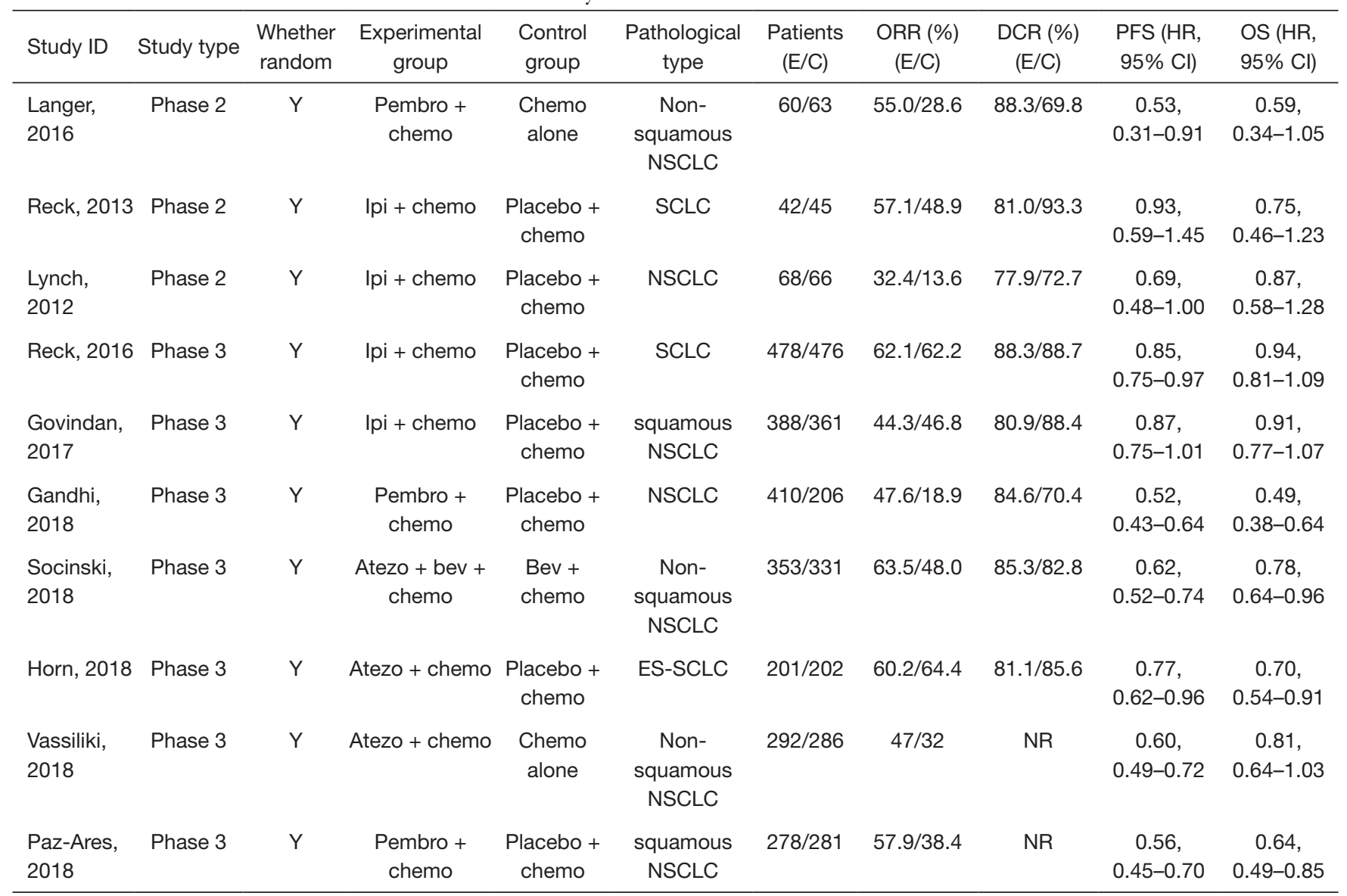

Y, yes; N, no; E, experimental; C, control; pembro, pembrolizumab; nivo, nivolumab; atezo, atezolizumab; bev, bevacizumab; ipi, ipilimumab; chemo, chemotherapy; NSCLC, non-small cell lung cancer; ES-SCLC, extensive-stage small cell lung cancer; ORR, objective response rate; DCR, disease control response; PFS, progression-free survival; OS, overall survival; HR, hazard ratio; Cl, confidence interval; NR, not reported.

Random sequences were generated by using the random number table in eight trials $(13,19,21,22,24-27)$, while random sequences were not generated in the other two trials $(20,23)$. Except for one trial (19), none of the trials provided detailed information about the allocation concealment. Blinding of participants and personnel was performed adequately in all studies. Blinding of outcome assessment was described in detail in three studies (21-23) and not clearly described in the remaining seven studies. The follow-up data were complete in all but one study (26). The selective reporting bias was low in four studies $(19,21,24,27)$ and not clear in the remaining six studies $(20,22,23,25,26)$. Other potential biases, such as specific study design, incomplete information and baseline unbalance were not clear in all ten studies. Overall, the methodological qualities of these studies were high. The details of bias risks assessment were summarized in Figures 2,3.

\section{Efficacy assessment}

Ten studies including 4,887 patients evaluated the efficacy of immunotherapy combined with chemotherapy, which was assessed in terms of the OR value (Table 2). The pooled ORs for ORR and DCR were 1.85 (95\% CI: 1.30-2.63, $\mathrm{P}<0.01)$ and 1.14 (95\% CI: $0.70-1.86, \mathrm{P}<0.01)$, respectively. Subgroup analysis revealed that the pooled ORs for ORR in PD-1/L1 blockade for NSCLC (subgroup A), CTLA4 blockade for SCLC (subgroup B), and CTLA-4 blockade for NSCLC (subgroup C) were 2.36 (95\% CI: 1.79-3.13, $\mathrm{P}<0.001), 1.03$ (95\% CI: $0.80-1.32, \mathrm{P}=0.832$ ) and 1.54 (95\% CI: 0.48-4.99, $\mathrm{P}=0.472$ ), respectively (Figure $4 A$ ). The pooled ORs for DCR in subgroup A, subgroup B and 
subgroup C were 1.92 (95\% CI: $1.10-3.35, \mathrm{P}<0.001), 0.66$ (95\% CI: $0.23-1.92, \mathrm{P}=0.832$ ) and 0.75 (95\% CI: 0.37-1.52, $\mathrm{P}=0.472$ ), respectively (Figure $4 B$ ). The random effects model was adopted when significant heterogeneity was observed among the studies $\left(\mathrm{I}^{2}=86.0 \%, \mathrm{P}_{\mathrm{Q}}=0.000\right)$.

\section{Prognosis evaluation}

We used the following two indicators to evaluate prognosis: PFS and OS (Table 2). The pooled HR value for PFS was 0.67 (95\% CI: $0.58-0.79, \mathrm{P}<0.001$ ). Subgroup analysis indicated that the pooled HRs for PFS in subgroup A, subgroup B and subgroup C were 0.58 (95\% CI: 0.52-0.63, $\mathrm{P}<0.001), 0.86$ (95\% CI: $0.76-0.97, \mathrm{P}<0.05)$ and $0.83(95 \%$ CI: $0.68-1.00, \mathrm{P}<0.05$ ), respectively (Figure $5 A$ ). When significant heterogeneity was detected, the random effects model was adopted $\left(\mathrm{I}^{2}=77.9 \%, \mathrm{P}_{\mathrm{Q}}=0.001\right)$. Moreover, the PD-1/L1 inhibitors plus chemotherapy for NSCLC group was further divided based on PD-L1 expression. The pooled HRs for PFS in the PD-L1 expression of $<1 \%, 1 \%$ to $49 \%$ and $\geq 50 \%$ groups were 0.66 (95\% CI: $0.52-0.83, \mathrm{P}<0.01$ ), 0.61 (95\% CI: 0.51-0.72, P<0.001) and 0.38 (95\% CI: 0.30 $0.47, \mathrm{P}<0.001$ ), respectively (Figure $6 A$ ). The random effects model was adopted when significant heterogeneity was detected among the studies $\left(\mathrm{I}^{2}=59.6 \%, \mathrm{P}_{\mathrm{Q}}=0.004\right)$. Subgroup analysis revealed similar results in OS. In the meanwhile, the pooled HRs for OS in subgroup A, subgroup B and subgroup $C$ were 0.67 (95\% CI: 0.55-0.81, $\mathrm{P}<0.001$ ), 0.92 (95\% CI: 0.80-1.06, $\mathrm{P}=0.266$ ) and 0.90 (95\% CI: 0.78-1.05, $\mathrm{P}=0.193$ ), respectively (Figure $5 B$ ). The random effects model was adopted when significant heterogeneity was observed $\left(\mathrm{I}^{2}=67.2 \%, \mathrm{P}_{\mathrm{Q}}=0.002\right)$. In addition, the combined HRs for OS in the PD-L1 expression $<1 \%, 1 \%$ to $49 \%$ and $\geq 50 \%$ groups were 0.60 (95\% CI: $0.43-0.83, \mathrm{P}<0.01$ ), 0.56 (95\% CI: $0.40-0.78, \mathrm{P}<0.01$ ) and 0.52 (95\% CI: $0.36-0.74$,
$\mathrm{P}<0.01$ ), respectively (Figure $6 B$ ). The fixed effects model was adopted when limited heterogeneity was observed $\left(\mathrm{I}^{2}=0 \%, \mathrm{P}_{\mathrm{Q}}=0.852\right)$. Besides, the pooled $\mathrm{HR}$ for OS without central nervous system (CNS) metastasis was 0.67 (95\% CI: $0.55-0.80, \mathrm{P}<0.001$ ) (Figure 7).

Thus, these results suggest a significant benefit of anti-

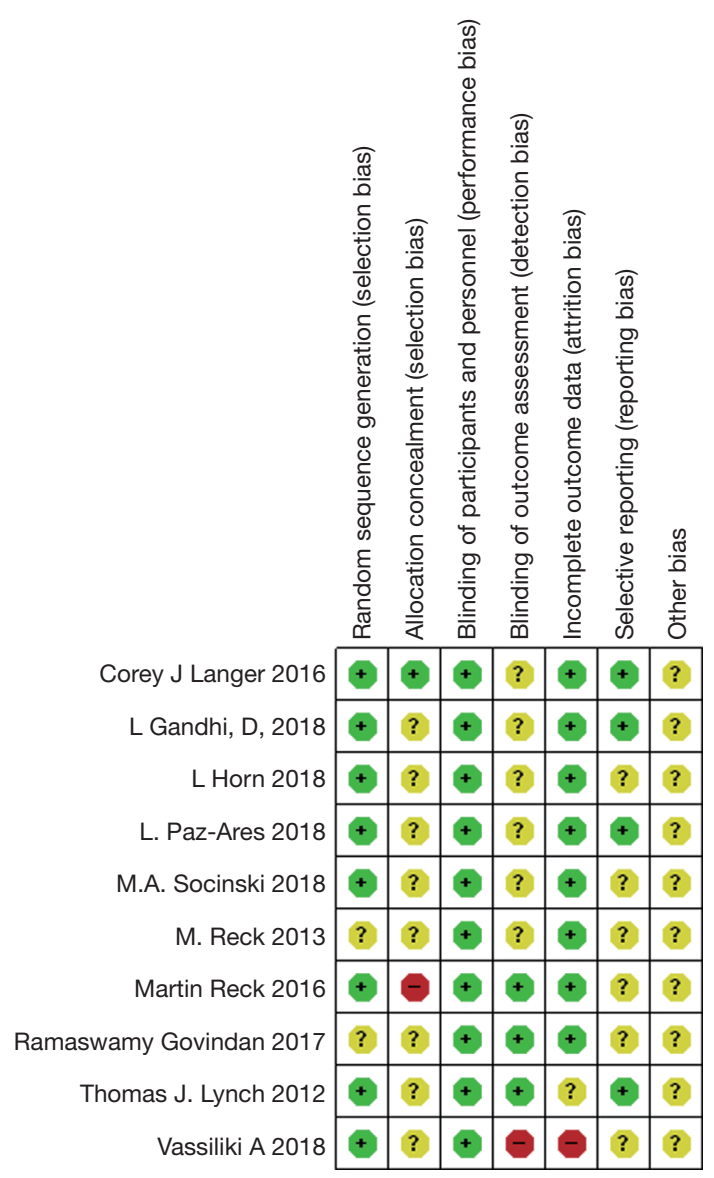

Figure 2 Risk of bias summary.

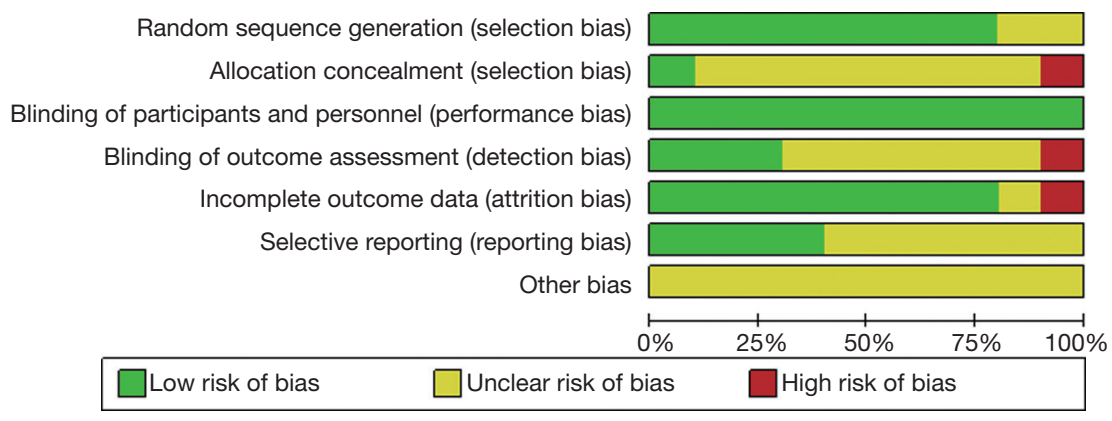

Figure 3 Risk of bias graph. 
Table 2 Subgroup analysis of ORR, DCR, PFS, OS, all TRAEs and 3 to 5 TRAEs

\begin{tabular}{|c|c|c|c|c|c|c|c|}
\hline Subgroup analysis & $\begin{array}{l}\text { No. of } \\
\text { studies }\end{array}$ & $\begin{array}{l}\text { Pooled OR/HR/RR } \\
(95 \% \mathrm{Cl})\end{array}$ & \multicolumn{2}{|c|}{ Significance test of OR/HR/RR } & \multicolumn{3}{|c|}{ Heterogeneity } \\
\hline \multicolumn{8}{|l|}{ ORR } \\
\hline Total & 9 & $1.85(1.30-2.63)$ & 3.41 & $<0.01$ & 86.0 & 56.94 & 0.000 \\
\hline Subgroup A & 5 & $2.36(1.79-3.13)$ & 6.01 & $<0.001$ & 61.5 & 10.40 & 0.034 \\
\hline Subgroup C & 2 & $1.54(0.48-4.99)$ & 0.72 & 0.472 & 85.1 & 6.72 & 0.010 \\
\hline \multicolumn{8}{|l|}{ DCR } \\
\hline Total & 7 & $1.14(0.70-1.86)$ & 3.41 & $<0.01$ & 86.0 & 56.94 & 0.000 \\
\hline Subgroup A & 3 & $1.92(1.10-3.35)$ & 6.01 & $<0.001$ & 61.5 & 10.40 & 0.034 \\
\hline \multicolumn{8}{|l|}{ PFS } \\
\hline Total & 9 & $0.67(0.58-0.79)$ & 5.01 & $<0.001$ & 77.9 & 36.26 & 0.001 \\
\hline Subgroup A & 5 & $0.58(0.52-0.63)$ & 11.25 & $<0.001$ & 0.0 & 2.01 & 0.733 \\
\hline Subgroup B & 2 & $0.86(0.76-0.97)$ & 2.47 & $<0.05$ & 0.0 & 0.14 & 0.706 \\
\hline Subgroup C & 2 & $0.83(0.68-1.00)$ & 1.98 & $<0.05$ & 24.0 & 1.32 & 0.251 \\
\hline \multicolumn{8}{|l|}{ PD-L1 expression } \\
\hline Total & 4 & $0.55(0.47-0.65)$ & 6.89 & 0.000 & 59.6 & 27.21 & 0.004 \\
\hline$<1 \%$ & 4 & $0.66(0.52-0.83)$ & 3.47 & $<0.01$ & 52.4 & 6.31 & 0.098 \\
\hline Subgroup B & 2 & $0.92(0.80-1.06)$ & 1.11 & 0.266 & 0.0 & 0.74 & 0.389 \\
\hline Subgroup C & 2 & $0.90(0.78-1.05)$ & 1.30 & 0.193 & 0.0 & 0.04 & 0.837 \\
\hline \multicolumn{8}{|l|}{ PD-L1 expression } \\
\hline Total & 2 & $0.56(0.46-0.68)$ & 5.82 & 0.000 & 0.0 & 1.98 & 0.852 \\
\hline$<1 \%$ & 2 & $0.60(0.43-0.83)$ & 3.11 & $<0.01$ & 0.0 & 0.01 & 0.920 \\
\hline $1-49 \%$ & 2 & $0.56(0.40-0.78)$ & 3.40 & $<0.01$ & 0.0 & 0.01 & 0.917 \\
\hline$\geq 50 \%$ & 2 & $0.52(0.33-0.82)$ & 2.80 & $<0.01$ & 37.0 & 1.59 & 0.208 \\
\hline \multicolumn{8}{|l|}{ CNS metastasis } \\
\hline Total & 4 & $0.63(0.51-0.79)$ & 4.05 & 0.000 & 52.8 & 10.59 & 0.060 \\
\hline Yes & 2 & $0.59(0.21-1.72)$ & 0.96 & 0.339 & 78.3 & 4.58 & 0.032 \\
\hline No & 4 & $0.67(0.55-0.80)$ & 4.35 & $<0.001$ & 35.6 & 4.66 & 0.198 \\
\hline
\end{tabular}

Table 2 (continued) 
Table 2 (continued)

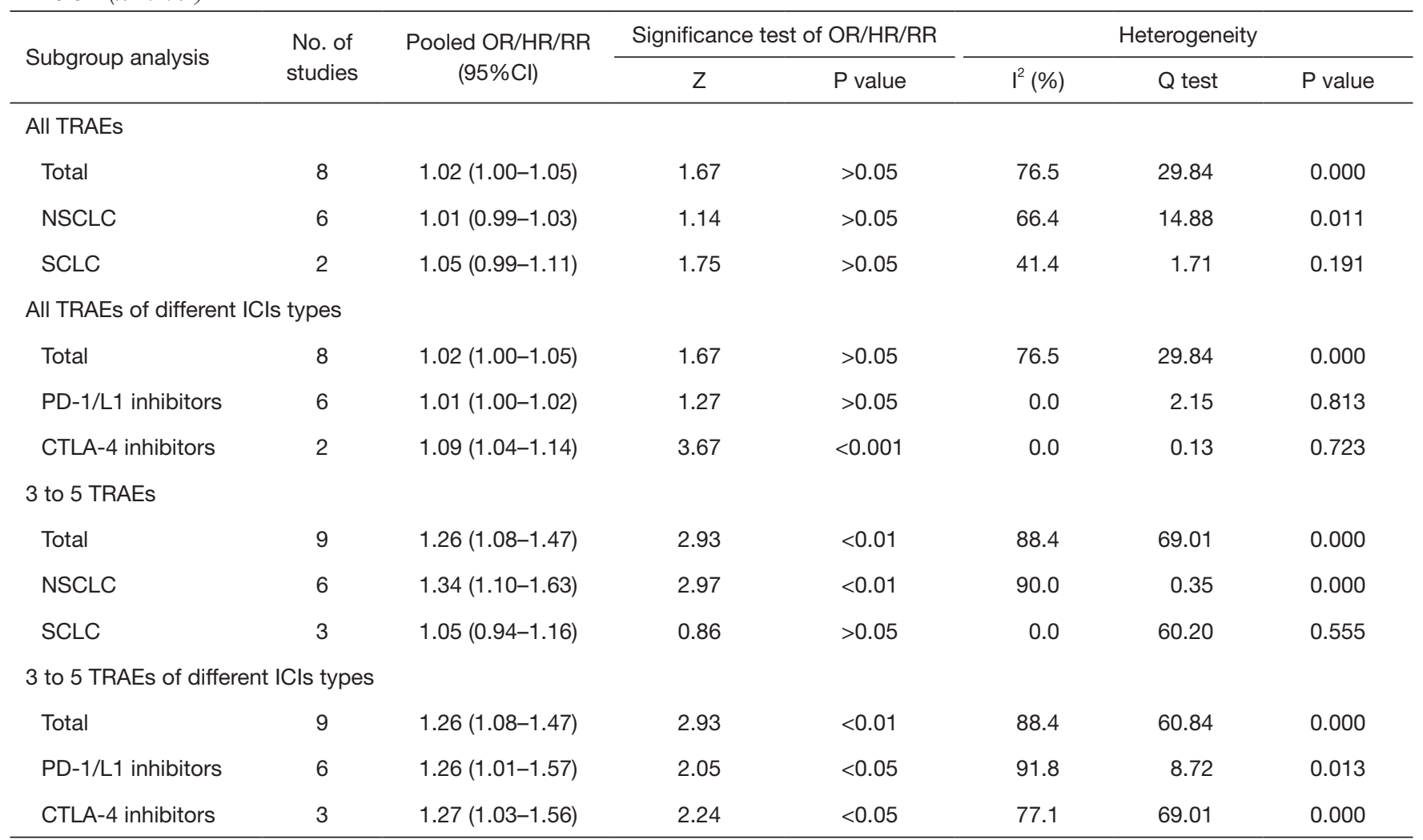

ORR, objective response rate; DCR, disease control response; PFS, progression-free survival; OS, overall survival; PD-1/L1, programmed cell death-1/L1; CTLA-4, cytotoxic T lymphocyte antigen-4; TRAEs, treatment-related adverse events; CNS, central nervous system; NSCLC, non-small cell lung cancer; SCLC, small cell lung cancer; OR, odds ratio; HR, hazard ratio; RR, relative risk; Cl, confidence interval; ICls, immune checkpoint inhibitors.

PD-1/L1 combined with chemotherapy for the treatment of NSCLC compared with chemotherapy alone, and as the level of PD-L1 expression increases, the benefit becomes more obvious. The prognosis seems better without CNS metastasis. Unfortunately, compared with chemotherapy alone, CTLA-4 blockade therapy combined with chemotherapy for the treatment of NSCLC or SCLC benefits only PFS and not OS.

\section{Safety assessment}

To evaluate the safety of PD-1/L1 or CTLA- 4 inhibitors combined with chemotherapy in lung cancer patients, TRAEs data were collected. Eight and nine studies provided the exact numbers of any grade and grade 3 to 5 TRAEs, respectively. The pooled RR for any grade TRAEs was 1.02 (95\% CI: $1.00-1.05, \mathrm{P}>0.05$ ). Subgroup analysis demonstrated that the RRs for NSCLC and SCLC were 1.01 (95\% CI: 0.99-1.03, P>0.05) and 1.05 (95\% CI: 0.99
1.11, P>0.05), respectively (Figure $8 A$ ). The pooled RRs for the PD-1/L1 and CTLA-4 blockades were 1.01 (95\% CI: 1.00-1.02, $\mathrm{P}>0.05$ ) and 1.09 (95\% CI: 1.04-1.14, $\mathrm{P}<0.001$ ), respectively (Figure $8 B$ ). The combined RR for grade 3 to 5 TRAEs was 1.26 (95\% CI: $1.08-1.47, \mathrm{P}<0.01$ ). Subgroup analysis revealed that RRs for NSCLC and SCLC were 1.34 (95\% CI: $1.10-1.63, \mathrm{P}<0.01)$ and 1.05 (95\% CI: 0.94-1.16, P>0.05), respectively (Figure $8 C$ ). Subgroup analysis based on the type of ICIs revealed that the RRs for the PD-1/L1 and CTLA-4 blockades were 1.26 (95\% CI: $1.01-1.57, \mathrm{P}<0.001)$ and 1.27 (95\% CI: $1.03-1.56, \mathrm{P}<0.05$ ), respectively (Figure $8 D$ ). All of above analyses were based on random effects models given that significant heterogeneity was observed.

The observed results indicated that the high levels of TRAEs caused by combination therapy regimens were significantly increased compared with those caused by chemotherapy alone, especially in the CTLA-4 inhibitor and NSCLC subgroup. 


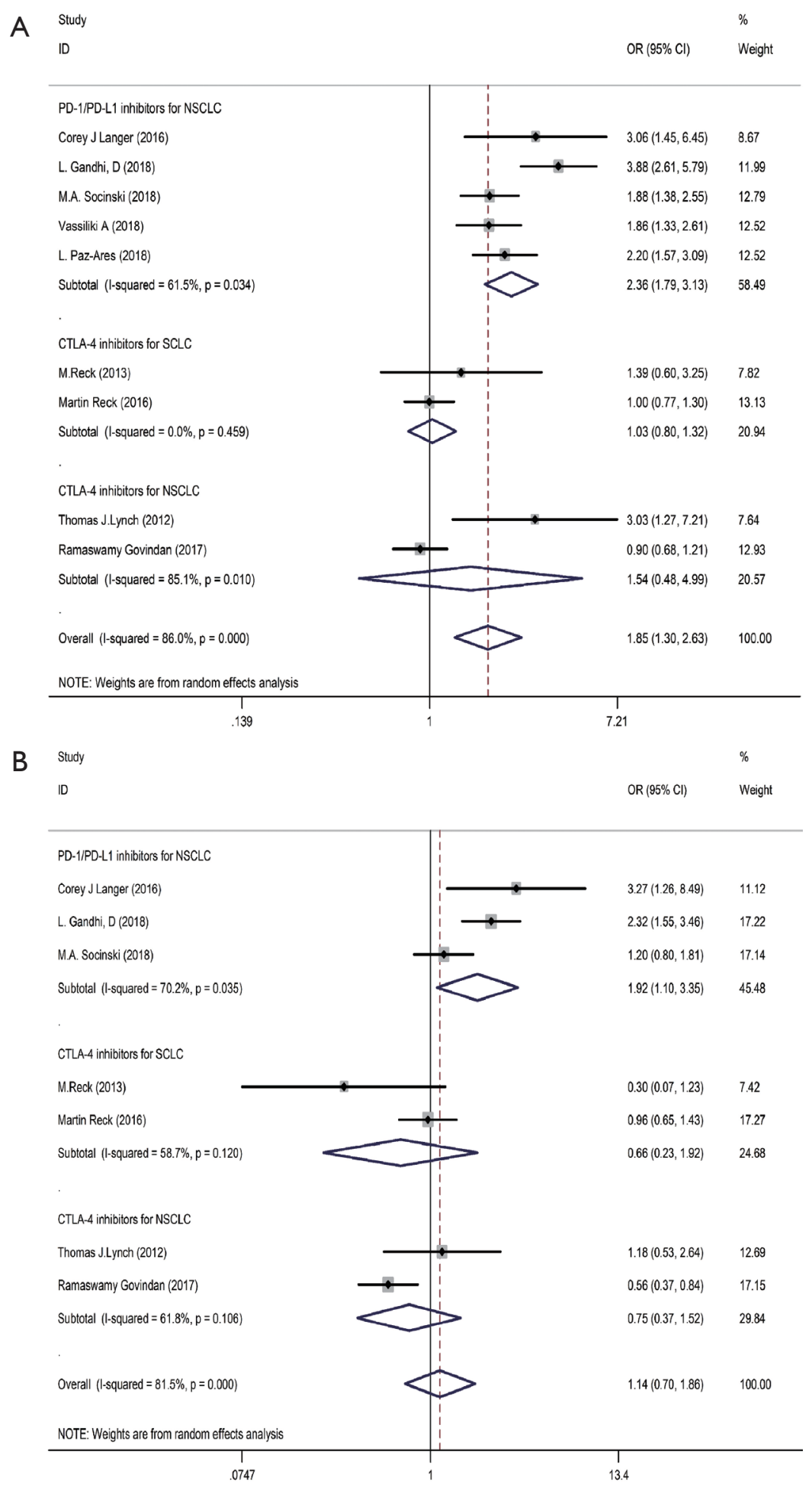

Figure 4 Meta-analysis of response rates in immunotherapy plus chemotherapy $v$ s. chemotherapy alone. (A) Forest plot of the pooled ORs for the ORR in subgroup A, subgroup B and subgroup C; (B) Forest plot of the pooled ORs for the DCR in subgroup A, subgroup B and subgroup C. Subgroup A, PD-1/L1 blockade for NSCLC; Subgroup B, CTLA-4 blockade for SCLC; Subgroup C, CTLA-4 blockade for NSCLC; PD-1/L1, programmed cell death-1/L1; CTLA-4, cytotoxic T lymphocyte antigen-4; NSCLC, non-small cell lung cancer; SCLC, small cell lung cancer; OR, odds ratio; ORR, objective response rate; DCR, disease control response. 


A Study
ID

B Study

ID

$\operatorname{HR}(95 \% \mathrm{Cl}) \quad$ Weight

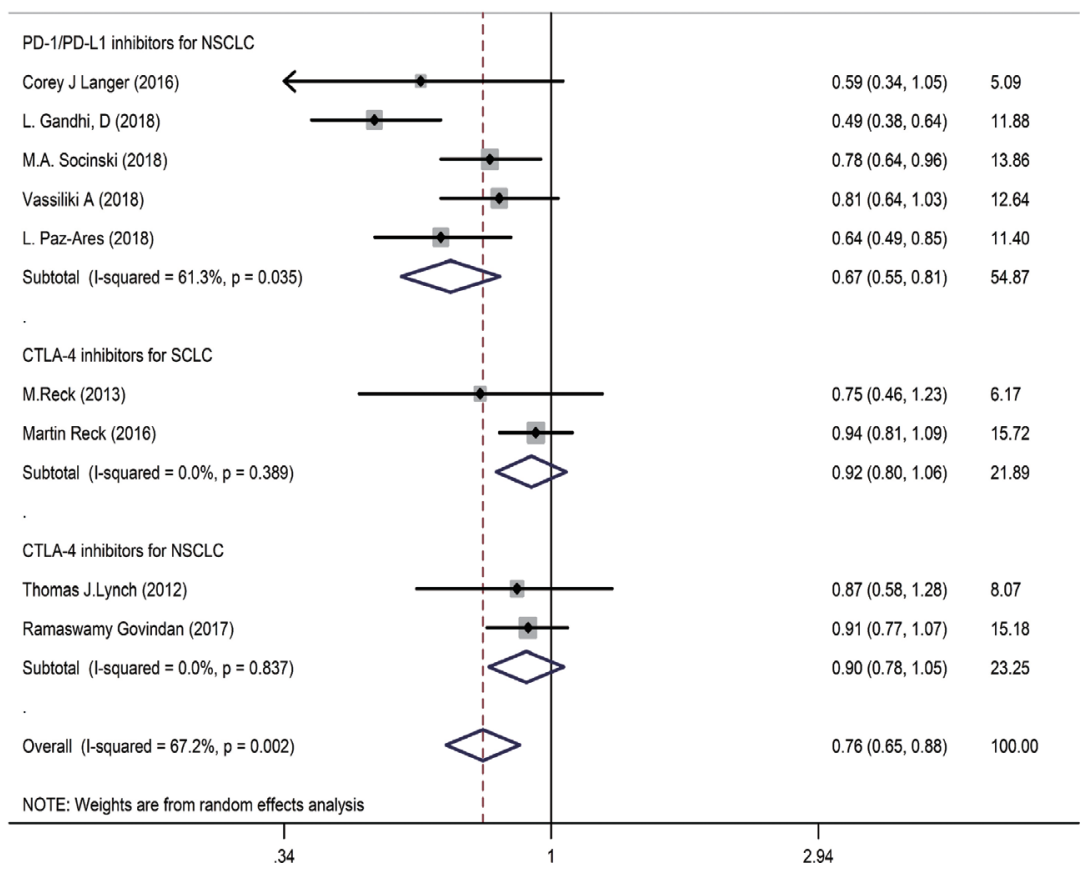

Figure 5 Meta-analysis of survival in immunotherapy plus chemotherapy $v$ s. chemotherapy alone. (A) Forest plot of the pooled HRs and its 95\% CIs for PFS in subgroup A, subgroup B and subgroup C; (B) forest plot of the pooled HR and 95\% CI for OS in subgroup A, subgroup B and subgroup C. Subgroup A, PD-1/L1 blockade for NSCLC; Subgroup B, CTLA-4 blockade for SCLC; Subgroup C, CTLA4 blockade for NSCLC; PD-1/L1, programmed cell death-1/L1; CTLA-4, cytotoxic T lymphocyte antigen-4; NSCLC, non-small cell lung cancer; SCLC, small cell lung cancer; PFS, progression-free survival; OS, overall survival; HR, hazard ratio; CI, confidence interval. 


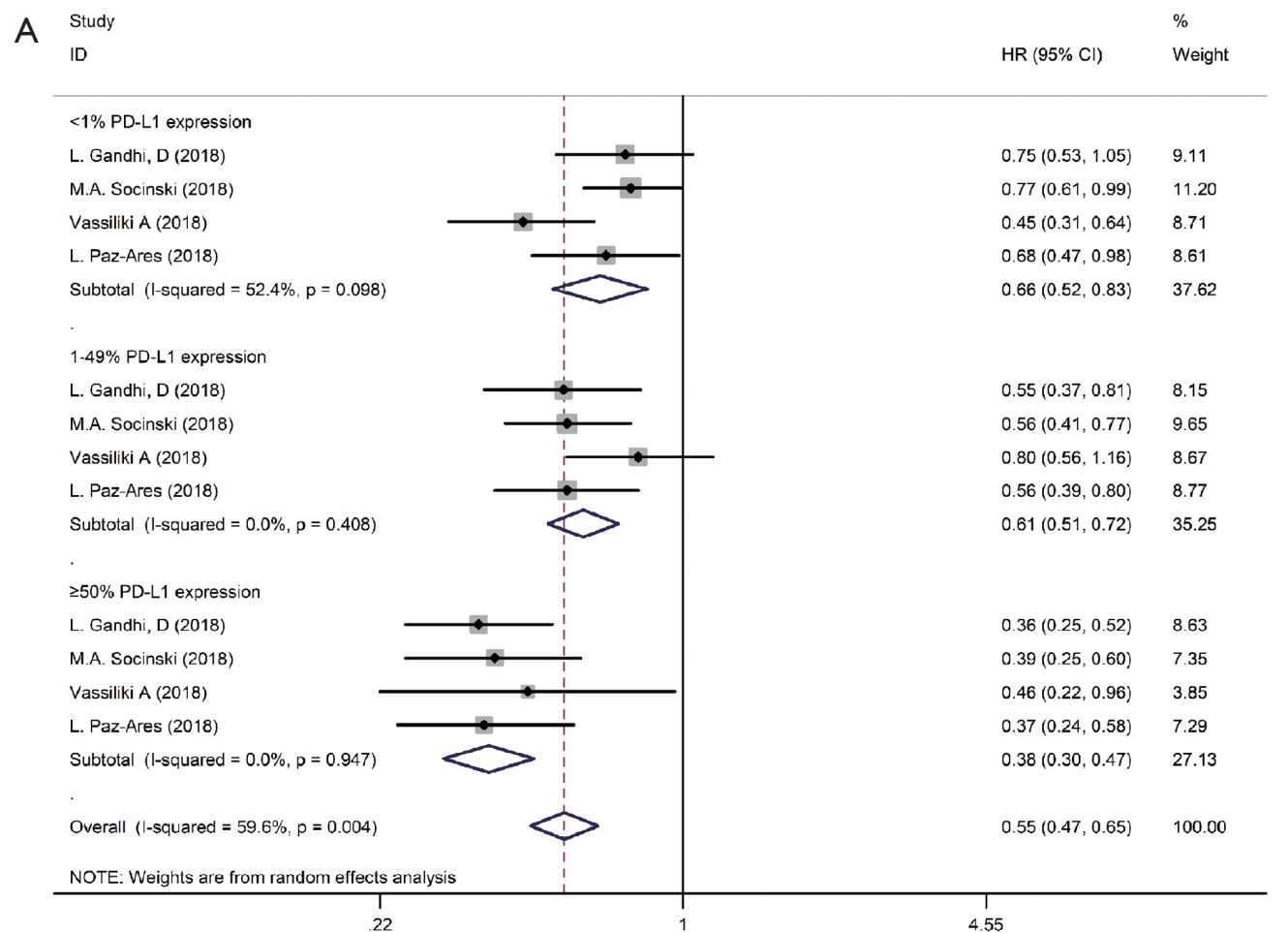

B study

ID

$\%$

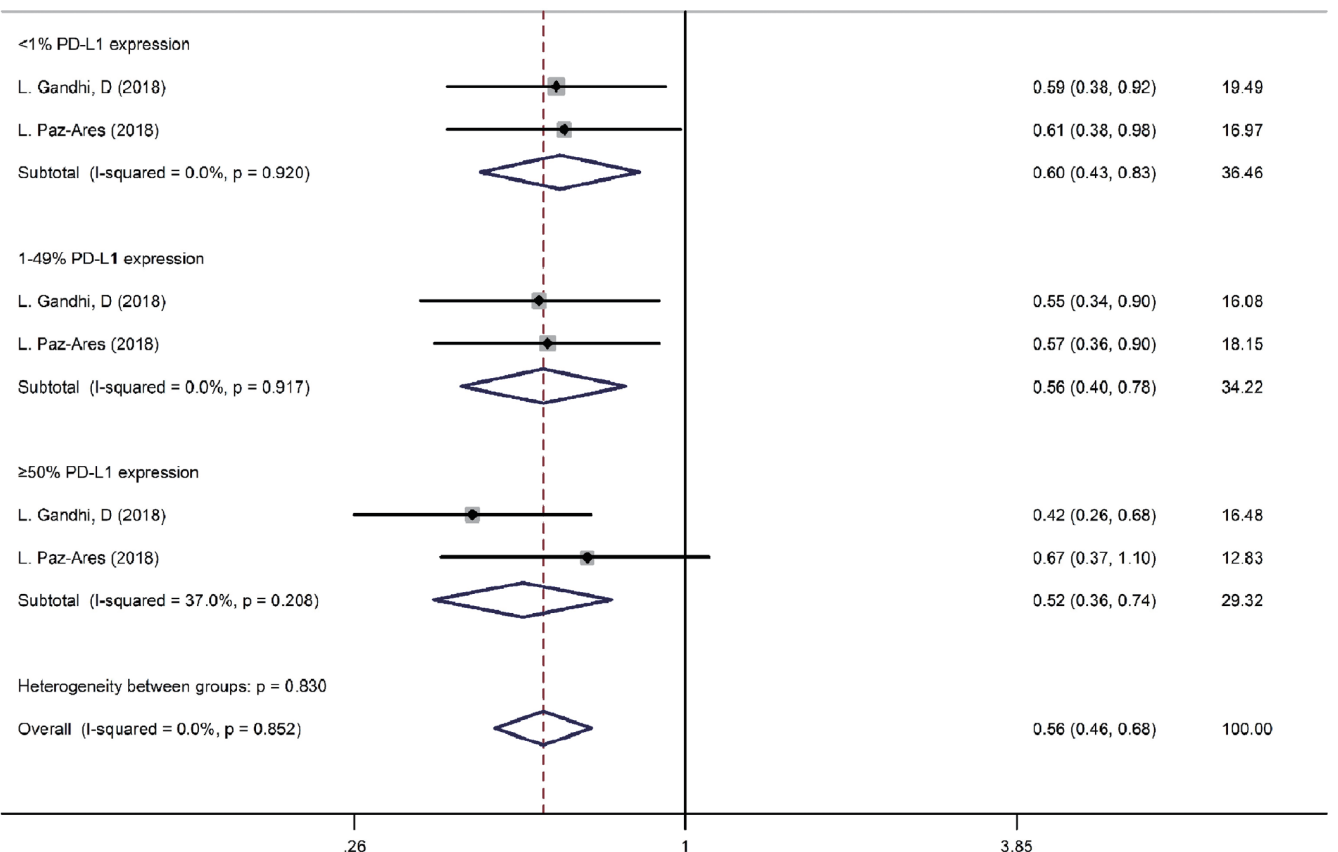

Figure 6 Meta-analysis of survival among different PD-L1 expression levels in immunotherapy plus chemotherapy vs. chemotherapy alone. (A) Forest plot of the pooled HRs and its $95 \%$ CIs for PFS in subgroup A with PD-L1 expression levels $<1 \%, 1 \%$ to $49 \%$ and $\geq 50 \%$; (B) forest plot of the pooled HRs and its 95\% CI for OS in subgroup A with PD-L1 expression levels < $1 \%$, $1 \%$ to $49 \%$ and $\geq 50 \%$. Subgroup A, PD-1/L1 blockade for NSCLC; PD-1/L1, programmed cell death-1/L1; NSCLC, non-small cell lung cancer; HR, hazard ratio; CI, confidence interval; PFS, progression-free survival; OS, overall survival. 


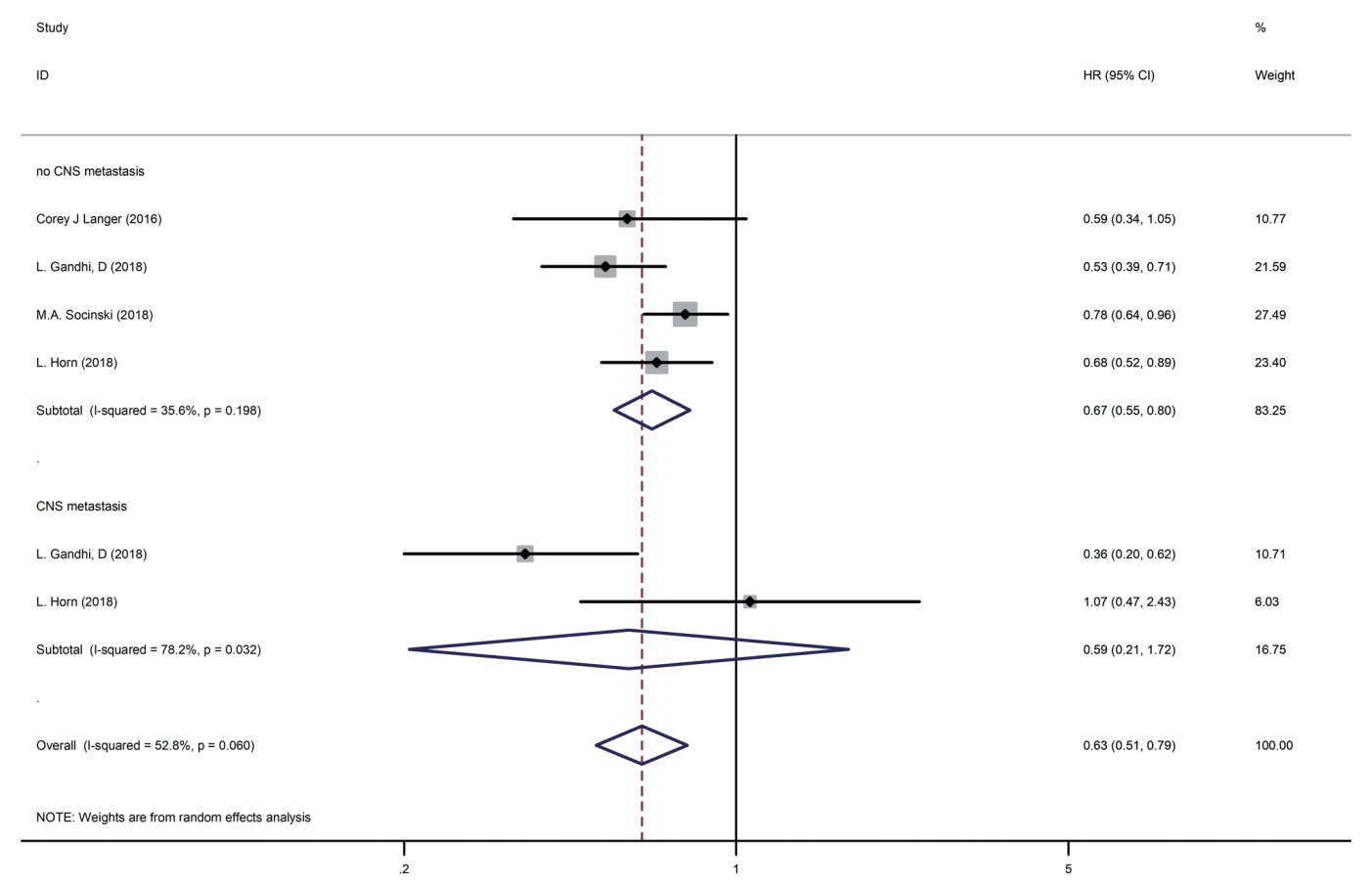

Figure 7 Forest plot of the pooled HR and its 95\% CI for OS in CNS metastasis subgroup. HR, hazard ratio; CI, confidence interval; OS, overall survival; CNS, central nervous system.

\section{Sensitivity analysis and publication bias}

Given that not all of the efficacy parameters were presented in all of the reviewed studies, we performed sensitivity analysis to detect the impacts of uncertain factors based on alternative study exclusion criteria. Our results were similar to those obtained from the overall analysis of the pooled trials upon the omission of any single study.

Next, to evaluate publication bias, Egger's funnel plots were constructed. Figure 9 shows no evidence of obvious asymmetry for ORR $(\mathrm{P}>|\mathrm{t}|=0.196$, Figure $9 A)$ or DCR $(\mathrm{P}>|\mathrm{t}|=0.980$, Figure $9 B)$. Similarly, regarding PFS and OS, there was no evidence for significant publication bias related to $\mathrm{PFS}(\mathrm{P}>|\mathrm{t}|=0.340$, Figure $10 A)$ or $\mathrm{OS}(\mathrm{P}>|\mathrm{t}|=0.138$, Figure 10B). In addition, there was also no publication bias for TRAE data based on the visual distribution of the funnel plot (data not shown), most likely because this meta-analysis included a limited number of studies.

\section{Discussion}

Rapid progression or lower ORRs after standard cytotoxic chemotherapy or immunotherapy alone for lung cancer patients indicates that a new effective therapeutic regimen is urgently needed. Currently, chemotherapeutic drugs can activate the tumor immune response via a variety of mechanisms to eradicate residual tumor cells, revealing the synergistic effect of chemotherapy and immunotherapy. For instance, chemotherapy can directly stimulate the congenital immune response and acquired immune cells (28), blocking the immunosuppressive pathway of tumor progression $(29,30)$ and enhancing the antigenicity and immunogenicity of tumor cells (31-33). We performed this meta-analysis to investigate the efficacy and safety of PD-1/ L1 or CTLA4 inhibitors combined with chemotherapy as a first-line treatment for lung cancer.

Some clinical trials assessed immunotherapy combined with chemotherapy in lung cancer patients. We observed significantly increased ORRs, DCRs, PFS and OS in treatment-naive patients who were treated with $\mathrm{PD}-1 / \mathrm{L} 1$ or CTLA-4 blockade therapy combined with chemotherapy compared with patients who received chemotherapy alone. To the best of our knowledge, our meta-analysis provided vigorous evidence of and insight into the efficacy and risks associated with using immunotherapy and chemotherapy in combination.

ICIs combined with chemotherapy showed synergistic effects in some preclinical trials $(34,35)$. Our research 

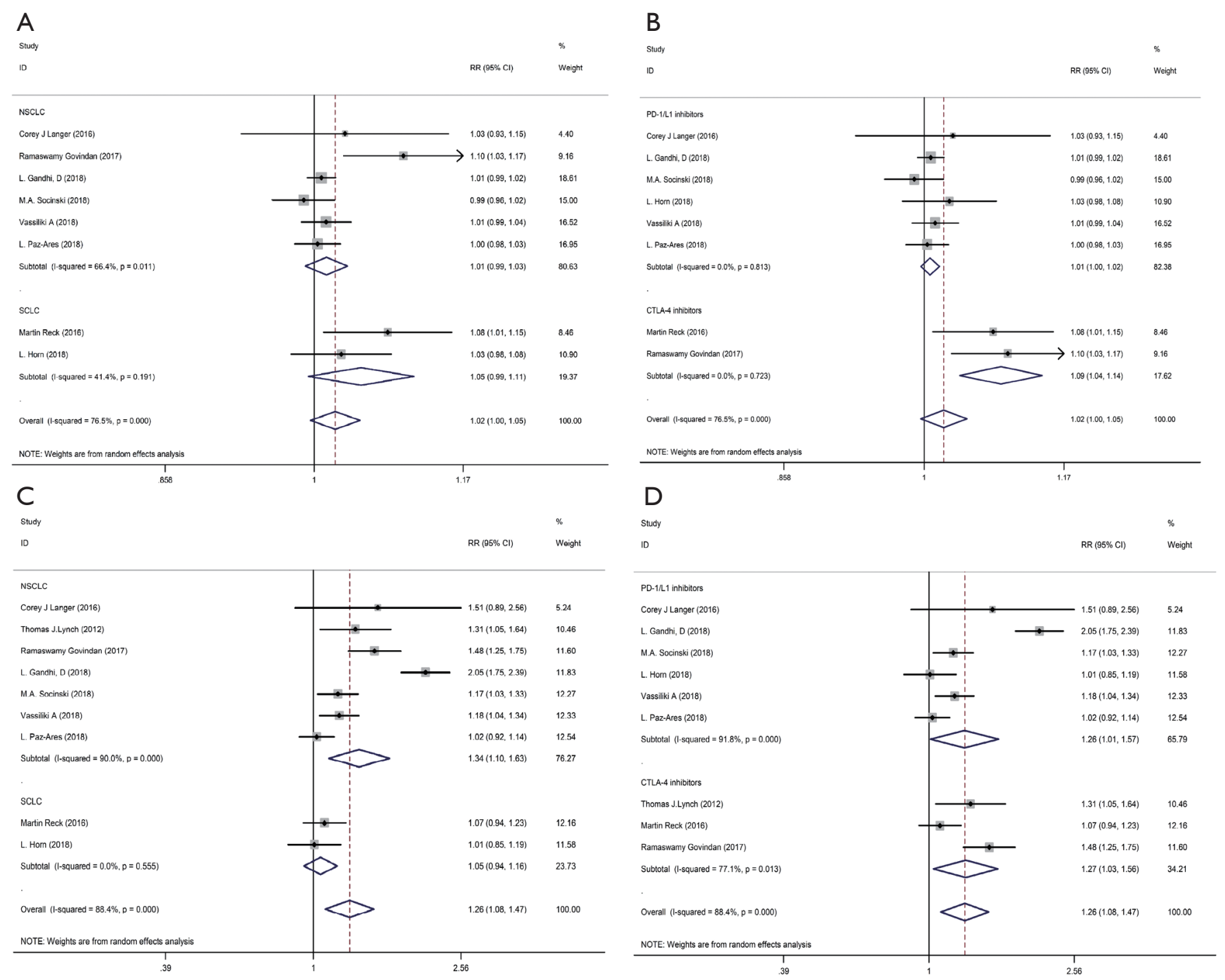

Figure 8 Meta-analysis of toxicity in immunotherapy plus chemotherapy vs. chemotherapy alone. (A) Forest plot of the pooled RRs for any grade TRAEs for immunotherapy combined with chemotherapy in NSCLC and SCLC; (B) forest plot of the pooled RRs for any grade TRAEs for PD-1/L1 inhibitors and CTLA-4 inhibitors combined with chemotherapy; (C) forest plot of the pooled RRs for grade 3 to 5 TRAEs for immunotherapy combined with chemotherapy in NSCLC and SCLC; (D) forest plot of the pooled RRs for grade 3 to 5 TRAEs for PD-1/L1 inhibitors and CTLA-4 inhibitors combined with chemotherapy. RR, relative risk; TRAEs, treatment-related adverse events; NSCLC, non-small cell lung cancer; SCLC, small cell lung cancer; PD-1/L1, programmed cell death-1/L1; CTLA-4, cytotoxic T lymphocyte antigen-4.

revealed an impressive efficacy of the combination of PD-1/L1 inhibitors with chemotherapy in treatmentnaive NSCLC patients, in which the pooled ORs for ORR and DCR were 2.36 (95\% CI: 1.79-3.31) and 1.92 (95\% CI: 1.10-3.35), respectively. Meanwhile, PFS and OS were significantly prolonged, with HRs of 0.58 (95\% CI: $0.52-0.63$ ) and 0.67 (95\% CI: 0.55-0.81), respectively. Xu et al. (36) performed a meta-analysis and demonstrated that ICIs plus chemotherapy significantly increased PFS but not OS in NSCLC. In our study, data regarding combined therapy were updated. We found that NSCLC patients administered combined therapy exhibited higher PFS and OS than patients treated with chemotherapy alone. One possible reason for this result was that more phase 3 studies 

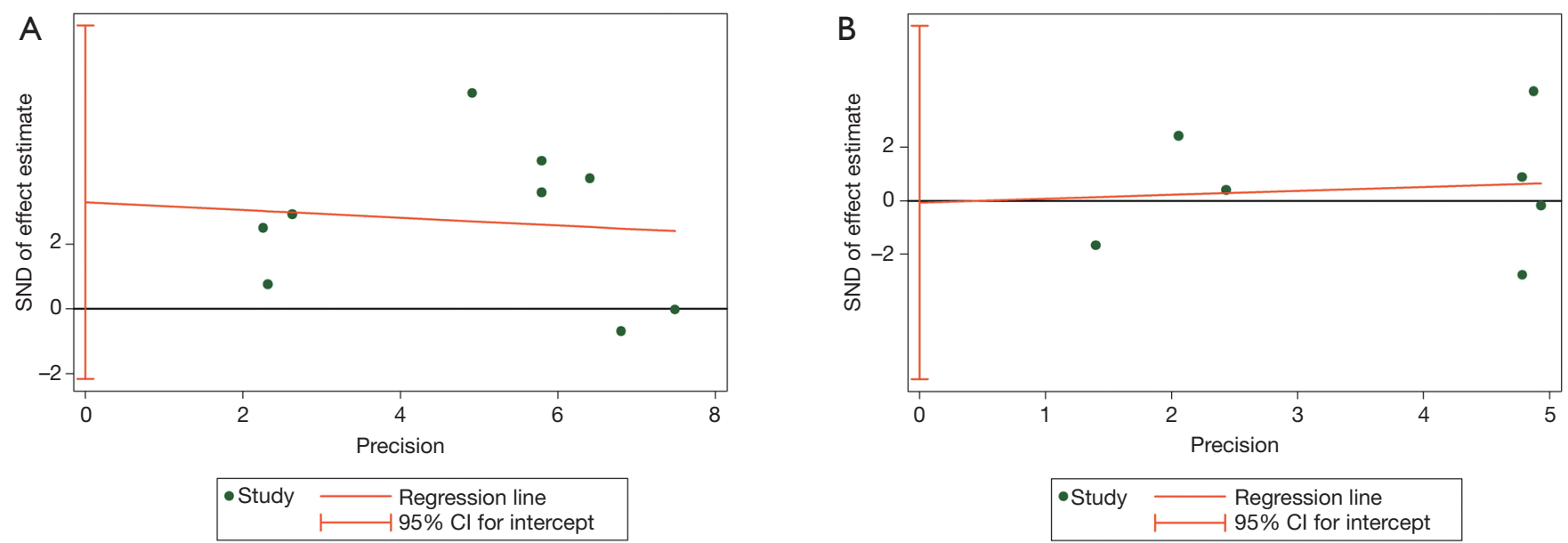

Figure 9 Publication bias of ORR and DCR. (A) Egger's funnel plot for all eligible studies that provided an OR for ORR; (B) Egger's funnel plot for all eligible studies that provided an OR for DCR. OR, odds ratio; ORR, objective response rate; DCR, disease control response.
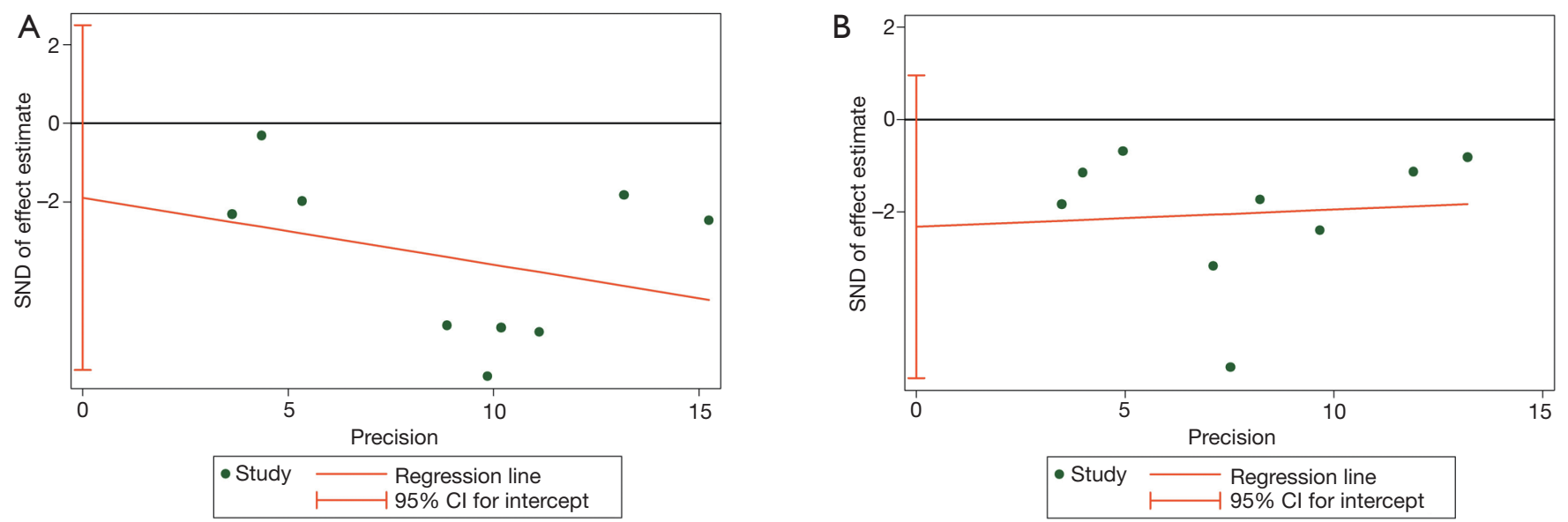

Figure 10 Publication bias of PFS and OS. (A) Egger's funnel plot for all eligible studies that provided a HR for PFS; (B) Egger's funnel plot for all eligible studies that provided a HR for OS. HR, hazard ratio; PFS, progression-free survival; OS, overall survival.

were included in our meta-analysis. Thus, this result should be confirmed in future clinical trials.

PD-1 and PD-L1 serve as a coinhibitory signal by blocking $\mathrm{T}$ cells that could continue to function and attack cancer cells $(37,38)$. The KEYNOTE-001 study (39) showed that NSCLC patients expressing PD-L1 at high levels significantly benefited from pembrolizumab. KEYNOTE-010 (40) and KEYNOTE-024 (41) also showed that the expression of PD-L1 was crucial for immunotherapy efficacy. In our study, higher PD-L1 expression levels were correlated with a greater the benefit of combination therapy, and this level may be an effective factor in predicting the efficacy of PD-1/L1 inhibitor plus chemotherapy for the treatment of NSCLC. Furthermore, a meta-analysis of the CheckMate 017 and CheckMate 057 (42) study showed that patients with brain metastasis who used nivolumab had a longer median OS and fewer TRAEs than patients treated with docetaxel. A retrospective analysis showed that immunotherapy was equally effective in patients with/without brain metastasis. Our study revealed that patients who did not appear to have baseline brain metastases had better immune treatment. Therefore, further studies are needed to verify these results.

SCLC patients with a higher tumor mutation burden (TMB) seem to have exhibit a better prognosis (43), suggesting that SCLC patients are more likely to benefit 
from immunotherapy. However, our results suggest that CTLA-4 blockade therapy plus chemotherapy for the treatment of SCLC is less than ideal, as only a PFS benefit was observed. Some studies showed that the mutations of some genes, such as TP53, RB1, and PTEN, negatively regulate the immune response $(44,45)$. The tumorsuppressor genes TP53 and RB1 are mutated in the majority of patients with SCLC, while PTEN mutations are present in $10-18 \%$ of SCLC patients (45), which may weaken the effectiveness of immunotherapy. Additional larger clinical trials are needed to verify this conclusion.

Notably, the benefits of checkpoint inhibitors used simultaneously or sequentially in combination with chemotherapy remain controversial. One NSCLC study (21) revealed that the phased ipilimumab plus chemotherapy group exhibited improved PFS and OS compared with those in the control but not to those in the concurrent ipilimumab group. In addition, regarding extensive SCLC disease (20), phased ipilimumab but not concurrent ipilimumab also improved PFS and OS versus those in the control group. Therefore, the administration of immunotherapy combined with chemotherapy still needs to be further explored.

Previous studies revealed that patients receiving PD-1/ L1 axis inhibitors exhibit fewer TRAEs than those receiving CTLA-4 inhibitors, whereas patients receiving combination therapy exhibit more TRAEs than patients receiving either monotherapy $(46,47)$. The most commonly reported TRAEs were nausea, vomiting, anemia, constipation, fatigue, neutropenia, sepsis, thrombocytopenia, rash, pyrexia, pneumonitis, acute kidney injury, hepatitis and peripheral neuropathy. Fatigue is the most commonly reported TRAEs across studies using PD-1/L1 and CTLA-4 inhibitors, occurring in $16-37 \%$ and $42 \%$ of patients respectively (48). Hu et al. (49) discovered that the incidence of pneumonitis among patients receiving $\mathrm{PD}-1 / \mathrm{L} 1$ inhibitors was significantly increased compared with that among patients undergoing chemotherapy. Diarrhea and colitis are most common toxicities observed in patients treated with CTLA4 blockade therapy rather than PD-1/L1 inhibitors $(49,50)$. In our study, we observed a significantly increased RR for all grade TRAEs in the CTLA-4 inhibitor group, while no significant difference was noted in the PD-1/L1 inhibitor group. Notably, in NSCLC group, the PD-1/L1 inhibitor group and the CTLA-4 inhibitor group, we also observed increased grade 3 to 5 TRAEs for those receiving combined therapy. These results suggest that the combination of CTLA-4 inhibitors plus chemotherapy was associated with an increased risk of TRAEs compared with that associated with the combination of $\mathrm{PD}-1 / \mathrm{L} 1$ axis inhibitors plus chemotherapy. In addition, combined therapies were associated with a high level of TRAEs. Therefore, caution should be applied regarding the clinical application of these combinations, and large clinical trials are further needed to establish conclusive evidence.

However, the shortcomings of this meta-analysis should be noted. Firstly, the number of studies included in this analysis was insufficient, especially in terms of subgroup analysis. Relevant subgroups, such as population characteristics, previous treatment regimens and sensitive gene mutations should be added in subsequent studies. Secondly, potential publication biases, such as race and ethnicity, were likely present despite the lack of evidence obtained from our statistical tests and further exploration is needed. Finally, studies were limited to those published in English, which may have resulted in a potential language bias.

In conclusion, our meta-analysis revealed that immunotherapy plus chemotherapy is an effective option as a first-line treatment for lung cancer and slightly increases the high level of adverse reactions. Thus, physicians should engage in balanced discussions with their patients on the risks and benefits of treatment options for lung cancer.

\section{Acknowledgements}

Funding: This work was supported by National Natural Science Foundation of China (Grant No. 81572273); National Natural Science Foundation of China (Grant No. 81602015); Natural Science Foundation of Jiangsu Province (Grant No. BK20161386); National Natural Science Foundation of China (Grant No. 81772500).

\section{Footnote}

Conflicts of Interest: The authors have no conflicts of interest to declare.

Ethical Statement: This study was approved by the ethics committee of Jinling Hospital affiliated to Medical School of Nanjing University.

\section{References}

1. Siegel RL, Miller KD, Jemal A. Cancer Statistics, 2017. CA Cancer J Clin 2017;67:7-30.

2. Scagliotti GV, Parikh P, von Pawel J, et al. Phase III 
study comparing cisplatin plus gemcitabine with cisplatin plus pemetrexed in chemotherapy-naive patients with advanced-stage non-small-cell lung cancer. J Clin Oncol 2008;26:3543-51.

3. Katsuya Y, Horinouchi H, Asao T, et al. Expression of programmed death 1 (PD-1) and its ligand (PD-L1) in thymic epithelial tumors: Impact on treatment efficacy and alteration in expression after chemotherapy. Lung Cancer 2016;99:4-10.

4. Brahmer J, Reckamp KL, Baas P, et al. Nivolumab versus Docetaxel in Advanced Squamous-Cell Non-Small-Cell Lung Cancer. N Engl J Med 2015;373:123-35.

5. Herbst RS, Baas P, Kim DW, et al. Pembrolizumab versus docetaxel for previously treated, PD-L1-positive, advanced non-small-cell lung cancer (KEYNOTE-010): a randomised controlled trial. Lancet 2016;387:1540-50.

6. Reck M, Rodriguez-Abreu D, Robinson AG, et al. Pembrolizumab versus Chemotherapy for PD-L1Positive Non-Small-Cell Lung Cancer. N Engl J Med 2016;375:1823-33.

7. Hodi FS, O'Day SJ, McDermott DF, et al. Improved survival with ipilimumab in patients with metastatic melanoma. N Engl J Med 2010;363:711-23.

8. Herzberg B, Campo MJ, Gainor JF. Immune Checkpoint Inhibitors in Non-Small Cell Lung Cancer. Oncologist 2017;22:81-8.

9. Meyers DE, Bryan PM, Banerji S, et al. Targeting the PD-1/PD-L1 axis for the treatment of non-small-cell lung cancer. Curr Oncol 2018;25:e324-34.

10. Santabarbara G, Maione P, Rossi A, et al. Novel immunotherapy in the treatment of advanced non-small cell lung cancer. Expert Rev Clin Pharmacol 2016;9:1571-81.

11. Weiss GJ, Waypa J, Blaydorn L, et al. A phase Ib study of pembrolizumab plus chemotherapy in patients with advanced cancer (PembroPlus). Br J Cancer 2017;117:33-40.

12. Horinouchi H, Yamamoto N, Fujiwara Y, et al. Phase I study of ipilimumab in phased combination with paclitaxel and carboplatin in Japanese patients with non-small-cell lung cancer. Invest New Drugs 2015;33:881-9.

13. Horn L, Mansfield AS, Szczesna A, et al. First-Line Atezolizumab plus Chemotherapy in Extensive-Stage Small-Cell Lung Cancer. N Engl J Med 2018. [Epub ahead of print].

14. Peng W, Chen JQ, Liu C, et al. Loss of PTEN Promotes Resistance to T Cell-Mediated Immunotherapy. Cancer Discov 2016;6:202-16.

15. Kato S, Goodman A, Walavalkar V, et al. Hyperprogressors after Immunotherapy: Analysis of Genomic Alterations
Associated with Accelerated Growth Rate. Clin Cancer Res 2017;23:4242-50.

16. Moher D, Liberati A, Tetzlaff J, et al. Preferred reporting items for systematic reviews and meta-analyses: the PRISMA statement. PLoS Med 2009;6:e1000097.

17. Higgins JP, Thompson SG, Deeks JJ, et al. Measuring inconsistency in meta-analyses. BMJ 2003;327:557-60.

18. Higgins JP, Altman DG, Gotzsche PC, et al. The Cochrane Collaboration's tool for assessing risk of bias in randomised trials. BMJ 2011;343:d5928.

19. Langer CJ, Gadgeel SM, Borghaei H, et al. Carboplatin and pemetrexed with or without pembrolizumab for advanced, non-squamous non-small-cell lung cancer: a randomised, phase 2 cohort of the open-label KEYNOTE-021 study. Lancet Oncol 2016;17:1497-508.

20. Reck M, Bondarenko I, Luft A, et al. Ipilimumab in combination with paclitaxel and carboplatin as first-line therapy in extensive-disease-small-cell lung cancer: results from a randomized, double-blind, multicenter phase 2 trial. Ann Oncol 2013;24:75-83.

21. Lynch TJ, Bondarenko I, Luft A, et al. Ipilimumab in combination with paclitaxel and carboplatin as first-line treatment in stage IIIB/IV non-small-cell lung cancer: results from a randomized, double-blind, multicenter phase II study. J Clin Oncol 2012;30:2046-54.

22. Reck M, Luft A, Szczesna A, et al. Phase III Randomized Trial of Ipilimumab Plus Etoposide and Platinum Versus Placebo Plus Etoposide and Platinum in Extensive-Stage Small-Cell Lung Cancer. J Clin Oncol 2016;34:3740-8.

23. Govindan R, Szczesna A, Ahn MJ, et al. Phase III Trial of Ipilimumab Combined With Paclitaxel and Carboplatin in Advanced Squamous Non-Small-Cell Lung Cancer. J Clin Oncol 2017;35:3449-57.

24. Gandhi L, Rodriguez-Abreu D, Gadgeel S, et al. Pembrolizumab plus Chemotherapy in Metastatic NonSmall-Cell Lung Cancer. N Engl J Med 2018;378:2078-92.

25. Socinski MA, Jotte RM, Cappuzzo F, et al. Atezolizumab for First-Line Treatment of Metastatic Nonsquamous NSCLC. N Engl J Med 2018;378:2288-301.

26. Papadimitrakopoulou VA, Cobo M, Bordoni R, et al. IMpower132: PFS and Safety Results with $1 \mathrm{~L}$ Atezolizumab + Carboplatin/Cisplatin + Pemetrexed in Stage IV Non-Squamous NSCLC. WCLC 2018. Available online: https://www.roche.com/dam/jcr:4ab12ea5-e78b4e00-9287-bc48aee375af/en/WCLC_IMpower132.pdf

27. Paz-Ares L, Luft A, Vicente D, et al. Pembrolizumab plus Chemotherapy for Squamous Non-Small-Cell Lung Cancer. N Engl J Med 2018. [Epub ahead of print]. 
28. Ramakrishnan R, Assudani D, Nagaraj S, et al. Chemotherapy enhances tumor cell susceptibility to CTLmediated killing during cancer immunotherapy in mice. J Clin Invest 2010;120:1111-24.

29. Zhang L, Dermawan K, Jin M, et al. Differential impairment of regulatory $\mathrm{T}$ cells rather than effector $\mathrm{T}$ cells by paclitaxel-based chemotherapy. Clin Immunol 2008;129:219-29.

30. Guertin DA, Sabatini DM. An expanding role for mTOR in cancer. Trends Mol Med 2005;11:353-61.

31. Srivastava P. Roles of heat-shock proteins in innate and adaptive immunity. Nat Rev Immunol 2002;2:185-94.

32. Obeid M, Tesniere A, Ghiringhelli F, et al. Calreticulin exposure dictates the immunogenicity of cancer cell death. Nat Med 2007;13:54-61.

33. Pol J, Vacchelli E, Aranda F, et al. Trial Watch: Immunogenic cell death inducers for anticancer chemotherapy. Oncoimmunology 2015;4:e1008866.

34. Kim YH, Choi BK, Kim KH, et al. Combination therapy with cisplatin and anti-4-1BB: synergistic anticancer effects and amelioration of cisplatin-induced nephrotoxicity. Cancer Res 2008;68:7264-9.

35. Ménard C, Martin F, Apetoh L, et al. Cancer chemotherapy: not only a direct cytotoxic effect, but also an adjuvant for antitumor immunity. Cancer Immunol Immunother 2008;57:1579-87.

36. Xu X, Huang Z, Zheng L, et al. The efficacy and safety of anti-PD-1/PD-L1 antibodies combined with chemotherapy or CTLA4 antibody as a first-line treatment for advanced lung cancer. Int J Cancer 2018;142:2344-54.

37. Lu J, Ramirez RA. The Role of Checkpoint Inhibition in Non-Small Cell Lung Cancer. Ochsner J 2017;17:379-87.

38. Khan M, Lin J, Liao G, et al. Comparative analysis of immune checkpoint inhibitors and chemotherapy in the treatment of advanced non-small cell lung cancer: A meta-analysis of randomized controlled trials. Medicine (Baltimore) 2018;97:e11936.

39. Kang SP, Gergich K, Lubiniecki GM, et al. Pembrolizumab KEYNOTE-001: an adaptive study leading to accelerated approval for two indications and a companion diagnostic. Ann Oncol 2017;28:1388-98.

40. Herbst RS, Baas P, Perez-Gracia JL, et al. P2.41 (also presented as PD1.06): Pembrolizumab vs Docetaxel for Previously Treated NSCLC (KEYNOTE-010): Archival vs New Tumor Samples for PD-L1 Assessment: Track: Immunotherapy. J Thorac Oncol 2016;11:S242-3.

41. Brahmer JR, Rodriguez-Abreu D, Robinson AG, et al. Health-related quality-of-life results for pembrolizumab versus chemotherapy in advanced, PD-L1-positive NSCLC (KEYNOTE-024): a multicentre, international, randomised, open-label phase 3 trial. Lancet Oncol 2017;18:1600-9.

42. Horn L, Spigel DR, Vokes EE, et al. Nivolumab Versus Docetaxel in Previously Treated Patients With Advanced Non-Small-Cell Lung Cancer: Two-Year Outcomes From Two Randomized, Open-Label, Phase III Trials (CheckMate 017 and CheckMate 057). J Clin Oncol 2017;35:3924-33.

43. Hellmann MD, Callahan MK, Awad MM, et al. Tumor Mutational Burden and Efficacy of Nivolumab Monotherapy and in Combination with Ipilimumab in Small-Cell Lung Cancer. Cancer Cell 2018;33:853-861.e4.

44. Shibata T, Kokubu A, Tsuta K, et al. Oncogenic mutation of PIK3CA in small cell lung carcinoma: a potential therapeutic target pathway for chemotherapy-resistant lung cancer. Cancer Lett 2009;283:203-11.

45. Tsoukalas N, Aravantinou-Fatorou E, Baxevanos P, et al. Advanced small cell lung cancer (SCLC): new challenges and new expectations. Ann Transl Med 2018;6:145.

46. Rittmeyer A, Barlesi F, Waterkamp D, et al. Atezolizumab versus docetaxel in patients with previously treated non-small-cell lung cancer (OAK): a phase 3, openlabel, multicentre randomised controlled trial. Lancet 2017;389:255-65.

47. Nagai H, Muto M. Optimal management of immunerelated adverse events resulting from treatment with immune checkpoint inhibitors: a review and update. Int J Clin Oncol 2018;23:410-20.

48. Palmieri DJ, Carlino MS. Immune Checkpoint Inhibitor Toxicity. Curr Oncol Rep 2018;20:72.

49. Hu YB, Zhang Q, Li HJ, et al. Evaluation of rare but severe immune related adverse effects in PD-1 and PD-L1 inhibitors in non-small cell lung cancer: a meta-analysis. Transl Lung Cancer Res 2017;6:S8-20.

50. Gupta A, De Felice KM, Loftus EV Jr, et al. Systematic review: colitis associated with anti-CTLA-4 therapy. Aliment Pharmacol Ther 2015;42:406-17.

Cite this article as: Shen K, Cui J, Wei Y, Chen X, Liu G, Gao X, Li W, Lu H, Zhan P, Lv T, Lin D. Effectiveness and safety of PD-1/PD-L1 or CTLA4 inhibitors combined with chemotherapy as a first-line treatment for lung cancer: A metaanalysis. J Thorac Dis 2018;10(12):6636-6652. doi: 10.21037/ jtd.2018.11.72 